\title{
Konzeptualisierung und empirische Überprüfung einer Progression zum Thema "Wirtschaftssektoren"
}

\author{
*anke.uhlenwinkel@aau.at, Institut für Geographie und Regionalforschung, Alpen-Adria-Universität Klagenfurt \\ ${ }^{* *}$ margrit.vohryzka@ph-kaernten.ac.at, Pädagogische Hochschule Kärnten \\ ***abrausam@edu.aau.at, Institut für Geographie und Regionalforschung, Alpen-Adria-Universität Klagenfurt \\ **** rumgeher@edu.aau.at, Institut für Geographie und Regionalforschung, Alpen-Adria-Universität Klagenfurt
}

eingereicht am: 01.10.2018, akzeptiert am: 09.07.2019

Im Kontext der Diskussion um mangelndes Wirtschaftswissen stellt der Beitrag die Frage, wie eine Progression des Verständnisses von wirtschaftlichen Sichtweisen und Modellen erreicht werden kann. Dazu werden zunächst verschiedene Ansätze der Progression theoretisch erörtert. Anschließend wird eine konzeptuell orientierte Progression für das Thema der Wirtschaftssektoren entworfen und empirisch getestet. Forschungsmethodisch wird dabei auf das Konzept der „lesson studies“ zurückgegriffen.

Keywords: Progression, Konzepte des GW-Unterrichts, lesson studies, Wirtschaftssektoren

\section{Conceptualisation and empirical testing of a progression relating to teaching "economic sectors"}

In the context of the debate on insufficient economic knowledge of students, this paper explores how a progression of the understanding of economic perspectives and models can be achieved over the span of several school years. First, different theoretical approaches to progression will be discussed. Subsequently, a conceptual progression relating to teaching economic sectors will be planned and empirically tested. The idea of "lesson studies" is used as research method.

Keywords: progression, geographical and economic concepts, lesson studies, economic sectors

\section{Einleitung}

Lange Zeit galt die Integration von Geographie und Wirtschaft in österreichischen Schulen auch als ein Garant für den Fortbestand des Faches Geographie. Damit unterschied sich Österreich von der Entwicklung in anderen Ländern, etwa in Deutschland, wo das Fach Geographie in vielen Bundesländern deutliche Stundenreduktionen hinnehmen musste.

Diese Situation hat sich in den letzten Jahren deutlich geändert. Einige Vertreter/innen der Wirtschaftspädagogik und der Wirtschaft generell fordern nun ein eigenes Fach Wirtschaft. Begründet wird dieses Ansinnen zum einen mithilfe von empirischen Studien, die auf der Inputseite belegen sollen, dass die GW-Schulbücher bei wirtschaftlichen Themen oftmals fehlerhaft seien und das Unternehmertum zu negativ darstellten (Haigner et al. 2016) oder auf der Outputseite zeigen, dass die Schüler/innen insgesamt über ein zu geringes Wirtschaftswissen verfügten (Fuhrmann et al. 2016). Zum anderen stellen gerade Vertreter/innen der Finanzwirtschaft einen Zusammenhang zwischen einem mangelndem Finanzwissen in der allgemeinen Bevölkerung und dem Ausbruch der Finanzkrise 2008 her: Die Banken haben aus ihren Fehlern gelernt und festgestellt, dass die Finanzausbildung an den Schulen eine „Katastrophe“ sei (Kleine Zeitung 28.10.2016). Gefordert wird dementsprechend eine eigene Finanzbildung, deren Resultat eine „financial literacy“ sein soll.

Dass dabei keine economic literacy, sondern lediglich eine eingeschränkte financial literacy gefordert wird, mag erstaunen, erschließt sich aber vermutlich aus der Schwerpunktsetzung der OECD, die ein entsprechendes Konzept bereits 2002 entwickelt hat (OECD 2013). Seit 2012 wird die financial literacy auch im 
Kontext der internationalen Vergleichsstudien des PISA-Konsortiums getestet. Das Ergebnis der ersten Studie ist allerdings wenig aufsehenerregend: Immerhin 10 Prozent der Schüler/innen erreichen Niveau 5 und können aus der PISA-Perspektive somit als finanziell gebildet angesehen werden. Dagegen bleiben 15 Prozent der Schüler/innen unter Niveau 2, was sie zu finanziellen Analphabetinnen und Analphabeten macht (OECD 2014: 54). Die Aussage, dass Schüler/ innen generell über zu wenig Wirtschaftswissen verfügten, wäre damit zumindest relativiert. Für Fragen der Progression interessanter als diese schlichte Situationsbeschreibung ist jedoch eine vergleichsweise ältere Literaturstudie von Willis (2008), die empirische Interventionsstudien zur Effektivität von Unterricht zur Förderung der angeblich fehlenden financial literacy gesichtet hat. Das Ergebnis ist niederschmetternd: Die wenigen vorhandenen Studien zeigen kaum oder sogar leicht negative Effekte (Willis 2008: 205), d. h. die Teilnehmer/innen sind nach dem Unterricht weniger kompetent in Finanzfragen, als sie es vorher waren.

Für Didaktiker/innen und Unterrichtspraktiker/ innen leitet sich aus diesen Erkenntnissen eine Frage $a b$, die sowohl für das „W“ als auch für das „ $G^{\prime \prime}$ im GW-Unterricht relevant ist: Wie können komplexe Inhalte über die Jahre hinweg so unterrichtet werden, dass 15-jährige potentielle PISA-Probandinnen und -Probanden mit diesem Wissen kompetent umgehen können? Oder anders: Wie muss Unterricht gestaltet sein, der, statt eine Abhandlung immer neuer Inhalte, die schnell vergessen werden, zu bieten, einen Aufbau von Wissen und Können initiiert?

Dieser Frage soll im Folgenden am Beispiel des Themas „Wirtschaftssektoren“ nachgegangen werden. Dazu werden unter Rückgriff auf die internationale Diskussion zunächst die bisherigen Überlegungen zur Progression im Geographieunterricht dargestellt. Anschließend wird die am Bundesrealgymnasium Viktring durchgeführte Pilotstudie vorgestellt.

\section{Das Konzept der Progression}

Das Adjektiv „progressiv“ ist Ende des 18. Jahrhunderts aus dem Französischen in die deutsche Sprache übernommen worden. Seine Bedeutung „stufenweise fortschreitend" bzw. „sich entwickelnd“ (DUDEN Herkunftswörterbuch) trifft noch heute den Kern seines didaktischen Verständnisses. Dementsprechend wird das Substantiv "Progression“ auch in seiner ersten Bedeutung als „Steigerung, Fortschreiten, Stufenfolge" (DUDEN Fremdwörterbuch) genutzt. Aus diesen drei Bedeutungen ergeben sich bereits die Gegenstände, auf die der Begriff der „Progression“ in der internationalen geographiedidaktischen Diskus- sion angewendet wird: Manche Beiträge legen einen Schwerpunkt auf die Betrachtung des Curriculums, d. h. auf den Lehrplan und in manchen Sprachkontexten auch auf die Unterrichtsplanung (Robertson et al. 2017). Festgelegt werden soll somit eine Stufenfolge. Andere Ausführungen stellen vornehmlich die Frage nach der Möglichkeit fachliche Inhalte schrittweise zu einem umfassenden Bild der Realität ausbauen zu können (Chang 2017), also eine Steigerung von Komplexität zu erreichen. Und im Rahmen der Diskussion um Kompetenzen wird in vielen Beiträgen vor allem die Progression von Fähigkeiten und Fertigkeiten thematisiert (Steegen et al. 2017), d. h. es wird ein Fortschreiten in der Entwicklung junger Menschen angestrebt. Die jeweilige Schwerpunktsetzung bedeutet in der Regel allerdings nicht, dass die Autorinnen und Autoren die anderen Aspekte völlig vernachlässigen. Oft ist ein multipler Ansatz zu beobachten. An dieser Stelle sollen nur die letzten beiden Verständnisse näher dargestellt werden, da sie für den empirischen Teil des Beitrags von besonderer Relevanz sind.

\subsection{Progression des fachlichen Inhalts}

Im deutschsprachigen Raum ist die Problematik der Progression vor allem zu Beginn der 1970er von Arnold Schultze thematisiert worden (BagolySimó \& Uhlenwinkel 2017). Dabei erweitert er die damalige akademisch-fachliche Diskussion um den Paradigmenwechsel weg von der Länderkunde und hin zur wissenschaftlich ergiebigeren allgemeinen Geographie um didaktische Argumente: Der länderkundliche Ansatz der Schulerdkunde böte kaum eine Möglichkeit zur Progression, da die Schüler/innen mit jedem neuen zu besprechenden Land wieder von vorne begännen. Einzelne Aspekte könnten kaum vertieft werden, weil anschließend bereits das nächste Land zu behandeln sei. Wenn ein solcher Unterricht überhaupt eine Progression böte, dann sei sie überaus flach. Im Rahmen der allgemeinen Geographie könne dagegen eine deutlichere Progression konzeptualisiert werden (Schmidt-Wulffen 1982). Schultzes eigenes allgemeingeographisch orientiertes Modell geht von einer Stufenfolge in vier Schritten aus, die er auf die Schuljahre 5 bis 10 verteilt. Jeder Schritt entspricht dabei einer didaktisch, nicht akademisch-fachlich begründeten Struktur: Begonnen wird mit den $\mathrm{Na}-$ turstrukturen, also jenen Gegenständen, die den Schülerinnen und Schülern natürliche Phänomene in verschiedenen Regionen zugänglich machen. Hieran schließt sich die Behandlung der Mensch-Naturstrukturen an, wobei Schultze an dieser Stelle in der praktischen Umsetzung meist auf die Beispiele sogenannter Naturvölker zurückgreift. Im Übergang vom 7. zum 8. Schuljahr kommen die funktionalen Struk- 
turen hinzu, mit denen die räumlichen Wirtschaftsverflechtungen etwa zwischen Rohstoffförderregionen und Regionen der industriellen Produktion dargestellt werden sollen. Ganz am Ende folgen die gesellschaftlich-kulturellen Strukturen, mit denen insbesondere die räumlich sichtbaren Ausdrücke politischer und gesellschaftlicher Vorstellungen thematisiert werden. Hierzu gehören etwa die absolutistische Straßenanlage in Karlsruhe oder sozialistische Prachtstraßen wie die Stalinallee / Karl-Marx-Allee in Ostberlin (Schultze 1970). Das Schicksal dieses Progressionsvorschlags ist äußerst ambivalent. Auf der theoretischen Ebene ist ihm sehr schnell eine Tendenz zu einem zumindest latenten Geodeterminismus vorgeworfen worden; ein Vorwurf den Schultze immer wieder von sich wies (ebd.). In der praktischen Arbeit als Schulbuchautor und -herausgeber hat Schultze sich selbst nur zum Teil an seinen eigenen Vorschlag gehalten, da er die erste und die zweite Struktur vertauscht hat, d. h. er hat mit den Mensch-Natur-Strukturen begonnen. Begründet hat er dieses Vorgehen nicht fachlich, sondern von der Schülerin bzw. vom Schüler her gedacht: 10- oder 11-Jährige interessierten sich eher für fremde Menschen und Lebensweisen als für pure Naturstrukturen. Während diese praktische Umsetzung seiner Vorstellung von inhaltlicher Progression durch das deutsche Schulbuch TERRA lang andauernde Auswirkungen aufdie Schulwirklichkeit hatte, ist die theoretische Diskussion um seinen Progressionsvorschlag schnell zum Erliegen gekommen. Nennenswerte neue Vorschläge für inhaltliche Progressionen hat es danach nicht mehr gegeben (Bagoly-Simó \& Uhlenwinkel 2017).

Im englischen Sprachraum und in Bezug auf didaktische Überlegungen insbesondere in Großbritannien ist die Diskussion etwas anders verlaufen. Zwar hat es auch hier den Paradigmenwechsel weg von der Länderkunde und hin zu einer wissenschaftlichen Kriterien gerecht werdenden Geographie gegeben, aber dieser Wandel ging mit einer intensiven Diskussion der Merkmale spezifisch geographischen Denkens einher. Die weitverzweigte Debatte kann und soll hier nicht wiedergegeben werden. Interessant im Kontext dieser Studie scheint aber eines ihrer Ergebnisse, das 2007 von Rawling entwickelt und bereits wenige Jahre später an verschiedenen Stellen aufgriffen wurde (Weeden $\&$ Butt 2009: 12). Es handelt sich dabei um den Versuch eine Progression mit Hilfe von geographischen Konzepten zu definieren. Die Progression beginnt aufgrund der Verteilung des Geographieunterrichts auf die britischen Schulstufen deutlich eher als Schultzes Progression, sie hört dafür aber auch etwas eher auf, d. h. sie fängt mit dem ersten Jahr der Grundschule an und endet bei etwa 14- bis 15-jährigen Schülerinnen und Schülern. Sie umfasst damit nicht nur sechs, sondern gut neun Schuljahre. Im ersten Schritt beschäfti- gen sich die Schülerinnen und Schüler mit kleinen, lokalen Orten, also dem Konzept "place“, das in diesem Kontext gut mit dem Hilfskonzept Wahrnehmung und Darstellung verbunden werden kann. Behandelt werden neben dem Heimatraum auch andere Räume weltweit. Dieser Zugang entspricht bis zu einem gewissen Grad Schultzes Mensch-Natur-Strukturen, nur wird er anders begründet und inhaltlich nicht mit Naturvölkern, sondern dem Leben von Kindern und jungen Menschen in der globalisierten Welt gefüllt. Gefolgt wird diese Stufe von einer Thematisierung der Kontexte, in denen sich die zuvor behandelten Orte entwickeln. Konzeptuell betrachtet wird die Vorstellung von place um das Konzept „space“ erweitert, also um die Interaktion zwischen den Orten. Auch hier können Parallelen zu Schultzes funktionalen Strukturen hergestellt werden, allerdings wiederum mit dem Hinweis, dass der britische Ansatz weniger von geographischen Phänomenen als vom geographischen Denken her begründet wird. $\mathrm{Zu}$ betonen ist auch noch einmal, dass die britischen Schüler/innen an diese Schritte deutlich eher herangeführt werden als die deutschen, weswegen die nächsten Schritte bei Schultze dann auch nicht mehr vorkommen. Im dritten Schritt werden im britischen Vorschlag explizit Orte auf verschiedenen Maßstabsebenen behandelt, um diese Maßstabsebenen im letzten Schritt miteinander zu verbinden und die Verflechtungen zwischen ihnen zu verstehen. Trotz der vorhandenen Ähnlichkeiten besteht der Vorteil dieser Form der Progression gegenüber dem Konzept von Schultze darin, dass sie sich tatsächlich auf gedankliche Strukturen, d. h. auf eine fachliche Perspektive, bezieht und nicht vor allem auf Phänomene, denen an sich eine fachliche Eigenschaft zugeschrieben wird. Mit der Fokussierung auf Konzepte und damit Denkakte ist der Gegenstand dem Ziel der Progression angemessen.

\subsection{Progression von Kompetenzen}

Mit der Veröffentlichung der Ergebnisse der ersten PISA-Studie im Jahr 2000 hat der Begriff „Kompetenzen" begonnen die pädagogischen und curricularen Diskussionen zu bestimmen. PISA definiert Kompetenz als „die Fähigkeit der Bewältigung komplexer Anforderungen, indem in einem bestimmten Kontext psychosoziale Ressourcen (einschließlich kognitive Fähigkeiten, Einstellungen und Verhaltensweisen) herangezogen und eingesetzt werden" (OECD 2005: 6) und weist zu den ersten drei untersuchten Kompetenzen - der Lesekompetenz, der mathematischen Kompetenz und der naturwissenschaftlichen Kompetenz - jeweils fünf Niveaustufen aus (als Beispiel siehe die Niveaustufen der mathematischen Kompetenz in Tab. 1). Obwohl diese Niveaustufen bei PISA nur dazu 
dienen, Leistungsunterschiede zu einem bestimmten Zeitpunkt zu messen, könnten sie auch, ähnlich wie im Gemeinsamen Europäischen Referenzrahmen für Sprachen (Europarat 2001), zur Beschreibung von Progressionen dienen.

Tab. 1: Kompetenzstufen der mathematischen Kompetenz bei PISA (Quelle: Stanat et al. 2002)

\begin{tabular}{|c|c|}
\hline $\begin{array}{l}\text { Kompe- } \\
\text { tenzstufe }\end{array}$ & Kompetenzbeschreibung \\
\hline 1 & $\begin{array}{l}\text { Schülerinnen und Schüler, die der Kompetenzstufe I } \\
\text { zugeordnet werden, verfügen lediglich über arith- } \\
\text { metisches und geometrisches Wissen auf Grund- } \\
\text { schulniveau. Sie können dieses Wissen abrufen und } \\
\text { unmittelbar anwenden, wenn die Aufgabenstellung } \\
\text { von vornherein eine bestimmte Standard-Mathema- } \\
\text { tisierung nahe legt. Sie sind aber überfordert, wenn } \\
\text { es darum geht, begriffliche Modellierungsleistungen } \\
\text { zu erbringen. }\end{array}$ \\
\hline 2 & $\begin{array}{l}\text { Auf der Kompetenzstufe II können einfachste begriff- } \\
\text { liche Modellierungen vorgenommen werden, die in } \\
\text { einen außermathematischen Kontext eingebettet } \\
\text { sind. Schülerinnen und Schüler auf dieser Stufe sind } \\
\text { in der Lage, unter mehreren möglichen Lösungsan- } \\
\text { sätzen den passenden zu finden, wenn durch Gra- } \\
\text { phiken, Tabellen, Zeichnungen usw. eine Struktur } \\
\text { vorgegeben ist, die das Modellieren erleichtert. Auch } \\
\text { auf dieser Stufe sind allerdings nur die Wissensin- } \\
\text { halte der Grundschulmathematik sicher verfügbar. }\end{array}$ \\
\hline 3 & $\begin{array}{l}\text { Von Stufe II zu Stufe III findet in mehrfacher Hin- } \\
\text { sicht ein qualitativer Sprung statt. Schülerinnen und } \\
\text { Schüler auf Stufe III verfügen auch über einfache } \\
\text { Wissensinhalte der Sekundarstufe I, also über den } \\
\text { Standardstoff der Lehrpläne aller Schulformen. Sie } \\
\text { können Konzepte aus unterschiedlichen mathema- } \\
\text { tischen Bereichen verknüpfen und zur Lösung von } \\
\text { Problemstellungen nutzen, wenn visuelle Darstellun- } \\
\text { gen den Lösungsprozess unterstützen. }\end{array}$ \\
\hline 4 & $\begin{array}{l}\text { Schülerinnen und Schüler auf Kompetenzstufe } \\
\text { IV bewältigen im technischen Bereich umfang- } \\
\text { reichere Verarbeitungsprozesse, können also } \\
\text { eine Lösung über mehrere Zwischenergebnisse } \\
\text { hinweg aufbauen. Auch offene Modellierungs- } \\
\text { aufgaben werden bewältigt, bei denen man } \\
\text { unter vielfältigen Lösungswegen einen eigenen } \\
\text { Ansatz finden muss. Verstärkt können auch } \\
\text { innermathematische begriffliche Zusammen- } \\
\text { hänge modelliert werden. }\end{array}$ \\
\hline 5 & $\begin{array}{l}\text { Auf der Kompetenzstufe } V \text { ist auch anspruchsvolles } \\
\text { curriculares Wissen verfügbar. Die Schülerinnen und } \\
\text { Schüler können sehr offen formulierte Aufgaben be- } \\
\text { wältigen, bei denen ein Modell frei gewählt bzw. } \\
\text { selbst konstruiert werden muss. Begriffliche Model- } \\
\text { lierungsleistungen auf dieser höchsten Stufe erfor- } \\
\text { dern häufig Begründungen und Beweise sowie das } \\
\text { Reflektieren über den Modellierungsprozess selbst. }\end{array}$ \\
\hline
\end{tabular}

Unabhängig davon, ob sie zur Beschreibung unterschiedlicher Leistungsniveaus zu einem Zeitpunkt oder zur Darstellung möglicher Progressionsstufen genutzt werden, weisen die inhaltlichen Ausführungen von Kompetenzstufen nahezu durchgehend zwei Probleme auf, die miteinander zusammenhängen. Das erste Problem besteht darin, dass unterstellt wird, dass in einem Feld erworbene Fähigkeiten und Fertigkeiten ohne Probleme auf ein anderes Feld transferiert werden können (Solem \& Lambert 2014). Das damit zusammenhängende Problem betrifft die Frage, ob und wenn ja, inwieweit - mögliche Progressionen von dem Vorhandensein von Vorwissen abhängen (Muñiz Solari, Solem \& Boehm 2017).

Die erst später von PISA entwickelten Kompetenzstufen zur oben bereits angesprochenen financial literacy scheinen insbesondere das letzte Problem zu berücksichtigen. Jedenfalls haben sie anders als die ursprünglichen drei Kompetenzen eine ausgeprägte Komponente deklarativen Wissens, was sie für die Betrachtung der Problematik der Formulierung von Progressionen in einem Sachfach interessant macht. Es werden folgende Kompetenzstufen (OECD 2014) definiert:

\section{Tab. 2: Kompetenzstufen der financial literacy} (eigene Darstellung nach OECD 2014)

\begin{tabular}{cl}
\hline $\begin{array}{l}\text { Kompe- } \\
\text { tenzstufe }\end{array}$ & Kompetenzbeschreibung \\
\hline 1 & $\begin{array}{l}\text { Die Schüler/innen können allgemeine Finanzpro- } \\
\text { dukte und -begriffe identifizieren und interpretieren. } \\
\text { Sie kennen den Unterschied zwischen Notwendig- } \\
\text { keiten und Wünschen und können einfache, all- } \\
\text { tägliche Kaufentscheidungen treffen. Sie können } \\
\text { einfache Rechenoperationen (Addition, Subtraktion, } \\
\text { Multiplikation) in finanziellen Kontexten anwenden. }\end{array}$ \\
\hline $\begin{array}{l}\text { Die Schüler/innen können ihr Wissen über allge- } \\
\text { meine Finanzprodukte und -begriffe anwenden. } \\
\text { Sie verstehen die Relevanz einer Buchführung. Sie } \\
\text { kennen die Relation zwischen Gebrauch und Kosten } \\
\text { einer Sache. Sie können einfache Rechenoperatio- } \\
\text { nen, inklusive der Division, in finanziellen Kontexten } \\
\text { anwenden. }\end{array}$ \\
$\begin{array}{l}\text { Die Schüler/innen können ihr Wissen über allge- } \\
\text { meine Finanzprodukte und -begriffe anwenden. } \\
\text { Sie können über die Auswirkungen von finanziellen } \\
\text { Entscheidungen nachdenken und entsprechend pla- } \\
\text { nen. Sie können einfache Rechenoperationen, inklu- } \\
\text { sive der Prozentrechnung, in finanziellen Kontexten } \\
\text { anwenden. Sie können selbst entscheiden, welche } \\
\text { Rechenoperation in welchen Kontexten relevant ist. }\end{array}$ \\
\hline $\begin{array}{l}\text { Die Schüler/innen können Wissen über we-- } \\
\text { niger bekannte Finanzprodukte und -begriffe } \\
\text { anwenden. Sie können mit dem Konzept Zin- } \\
\text { seszins kalkulieren. Sie können komplexere } \\
\text { Finanzdokumente verstehen. Sie können län- } \\
\text { gerfristige Auswirkungen von finanziellen Ent- } \\
\text { scheidungen, insbesondere bei der Aufnahme } \\
\text { von Krediten, überblicken. }\end{array}$ \\
$\begin{array}{l}\text { Die Schüler/innen können Wissen über eine große } \\
\text { Bandbreite verschiedener Finanzprodukte und -be- } \\
\text { griffe anwenden. Sie können bei der Analyse kom- } \\
\text { plexer Finanzprodukte auch solche Auswirkungen } \\
\text { bedenken, die in den Dokumenten nicht explizit ge- } \\
\text { nannten werden, etwa die Transaktionskosten. Sie } \\
\text { können die finanziellen Ergebnisse von weniger üb- } \\
\text { lichen Finanzentscheidungen genau abschätzen und } \\
\text { zeigen ein Verständnis für den weiteren finanziellen } \\
\text { Kontext, etwa hinsichtlich der Einkommenssteuer. }\end{array}$ \\
\hline $\mathbf{3}$
\end{tabular}

Schon ein Vergleich der Kompetenzstufen der mathematischen Kompetenz mit den Anforderungen an die Beherrschung von Rechenoperationen auf den einzelnen Kompetenzstufen der financial literacy 
zeigt einen deutlichen Unterschied in der Progression: Während die mathematischen Kompetenzstufen den Schwerpunkt auf ein immer differenzierteres mathematisches Verständnis legen, das am Ende zu einem selbstständigen Umgang mit der Materie führt, verfahren die Kompetenzstufenbeschreibungen der financial literacy weitgehend additiv: zunächst sind Addition, Subtraktion und Multiplikation gefragt, dann kommt die Division hinzu, auf der nächsten Kompetenzstufe die Prozentrechnung und am Ende die Zinseszinsrechnung. Auch die Nennung der selbständigen Wahl der Rechenoperationen auf Kompetenzstufe 3 kann den additiven Ansatz nicht verleugnen, denn sie scheint weitgehend kontingent. Würde sie zudem als völlig freie Entscheidung und nicht nur als eine Wahl aus einer vorgegebenen Menge von Möglichkeiten verstanden, dann wäre es eine Leistung, die die mathematische Kompetenz erst auf Kompetenzstufe 5 vorsieht. Lässt sich bei der Additivität der Rechenoperationen noch eine gewisse Progression erkennen, ist hinsichtlich der genannten deklarativen Wissensanteile nicht zu erkennen, wie die Progression zwischen den einzelnen Aspekten begründet werden soll. Es bleibt beispielsweise unklar, warum die Schüler/innen noch vor dem Verständnis der Einkommenssteuer über ein Verständnis der Kredite verfügen sollen.

Die Problematik der Definition der Kompetenzstufen der financial literacy liegt vor allem darin, dass dem fortschreitenden Verständnis weder allgemeine Fähigkeiten und Fertigkeiten wie bei der mathematischen Kompetenz noch fachliche Konzepte wie in der britischen geographiedidaktischen Diskussion zugrunde liegen. Letztlich geht die Kompetenzdefinition einen Schritt zurück und benennt lediglich finanzielle Phänomene, so ähnlich wie es in der deutschen geographiedidaktischen Diskussion der 1970er Jahre gemachtwurde.

Zusammenfassend kann festgehalten werden, dass sich die Bestimmung von Progressionen im schulischen Kontext sinnvollerweise entweder auf die Behandlung fachlicher Konzepte oder auf vergleichsweise allgemein formulierte überfachliche Konzepte bezieht. In den Sachfächern ergibt sich zusätzlich die Schwierigkeit, dass die Progression in einem dieser Bereiche über sehr unterschiedliche Lehrplaninhalte hinweg erkennbar sein muss. Es müsste dementsprechend gezeigt werden, wie das Thema "Gütererzeugung in gewerblichen und industriellen Betrieben " auf einer niedrigeren Kompetenzstufe unterrichtet wird als beispielsweise das Thema „Leben in einer vielfältigen Welt“, oder umgekehrt: welchen Kompetenzzuwachs das letztere Thema gegenüber dem ersteren erlaubt. Diese sehr anspruchsvolle Aufgabe haben wir in der im Folgenden dargestellten empirischen Studie umgangen, indem wir uns auf ein Thema konzentriert haben. Die Frage war damit zunächst nur, ob sich in einem Sachfach am Beispiel eines Themas eine Progression formulieren lässt und ob diese Progression die Lernmöglichkeiten und -bedürfnisse der Schüler/innen abbildet.

\section{Progression zum Thema ,Wirtschafts- sektoren“"}

Das Thema Wirtschaftssektoren wurde gewählt, weil es in der Schulbuchstudie von Haigner et al. (2016) als Beispiel für fehlerhafte inhaltliche Darstellungen im GW-Unterricht herangezogen wurde. Dabei konzentrieren sich die zuvor genannten Autoren auf die Darstellung des primären Sektors, der in sechs untersuchten Schulbüchern jeweils unterschiedlich definiert wurde: Während er in einem Buch nur die Landwirtschaft, die Forstwirtschaft und die Fischerei umfasse, zähle das nächste Schulbuch auch noch Jagd und Bergbau hinzu, und ein weiteres ließe die Fischerei einfach aus. Jedenfalls sei kein einheitliches Verständnis des primären Sektors in den Schulbüchern erkennbar. Diese Kritik ist zwar an sich schon wenig überzeugend, da die Schulbücher nur untereinander verglichen wurden, ohne dass eine Referenz von außen herangezogen worden wäre, um zu prüfen, welche Darstellung denn nun valide sei (Uhlenwinkel 2018), sie bleibt aber auch bei einem Gegenstandsverständnis stehen, das sich rein auf deklaratives Wissen bezieht und schon deswegen kaum eine Bestimmung von Progression zulässt. Dementsprechend soll hier kurz gezeigt werden, wie eine entsprechende Progression zum Thema aussehen könnte.

In der im Kontext dieser Pilotstudie getesteten Progression ist im ersten Schritt die Kategorisierung der Wirtschaftssektoren selbst thematisiert worden. Die Schüler/innen sollten sich mit den einzelnen Kategorien vertraut machen, indem sie bekannte Berufe zuordnen. Anschließend wurde das Konzept von Wahrnehmung und Darstellung ${ }^{1}$ am Beispiel des primären Sektors problematisiert. Zum einen führt die Kennzeichnung von Nahrungsmitteln als „, hergestellt in Österreich" zu dem Eindruck, dass es sich bei Österreich um ein Agrarland handeln könnte, in dem noch dazu Kakao angebaut wird (vgl. Abb. 1), und das Traditionen anderer Länder um eine Qualität ergänzt, die diese Länder selbst nicht erbringen können (vgl. Abb. 2). Zum anderen kann am Beispiel von Imbiss-PommesFrites gezeigt werden, dass an dem Prozess ihrer Herstellung alle drei Wirtschaftssektoren beteiligt sind. Werden mehrere dieser Schritte in einem Unternehmen durchgeführt, wird es schwierig, dieses Unternehmen einem einzigen Wirtschaftssektor zuzuschreiben.

Im Modell von Taylor (2008) handelt es sich hierbei um ein Hilfskonzept. Im österreichischen Lehrplan für die AHS-Oberstufe wird es als Basiskonzept angesehen. 

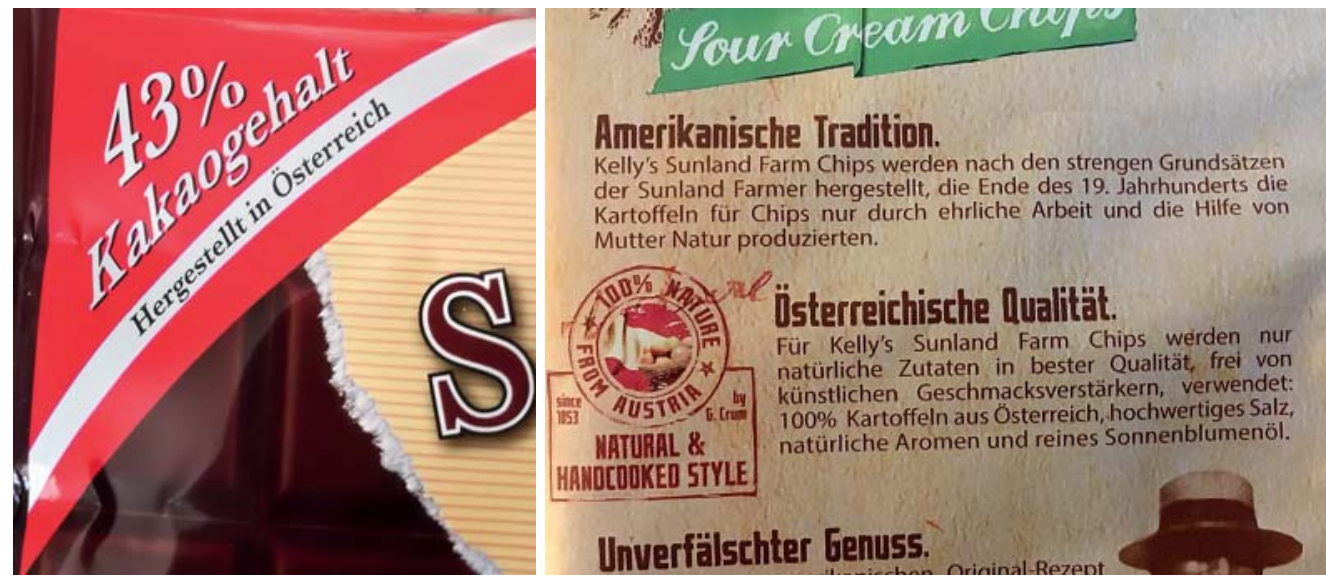

\author{
Abb. 1: Kakao aus \\ Österreich? \\ (Foto: Anna Brausam) \\ Abb. 2: Österreich als Teil \\ der USA? \\ (Foto: Anke Uhlenwinkel)
}

Im zweiten Schritt wird das Modell des sektoralen Wandels thematisiert. Am Beispiel einer Familie wird gezeigt, wie sich die Berufsprofile der Familienmitglieder über die Generationen verändern. Die dem Modell inhärente Modernisierungsvorstellung wird am Beispiel einzelner wirtschaftlicher Tätigkeiten in verschiedenen Ländern überprüft. Hierzu gehören etwa der exportorientierte Soja-Anbau in den USA, der hohe Anteil der Industrieproduktion in Liechtenstein oder die relativ kostengünstigen und gleichzeitig qualitativ hochwertigen Angebote indischer Krankenhäuser für Patientinnen und Patienten aus den westlichen Industrieländern.

Im letzten Schritt soll das Modell der Wirtschaftssektoren selbst differenziert werden. Dabei liegt das Augenmerk zum einem auf der mehrfach vorgeschlagenen und in wissenschaftlichen Kontexten auch genutzten Aufteilung des tertiären Sektors in einen tertiären (Dienstleistungen), quartären (Informationsdienstleistungen) und quintären (Entsorgungswirtschaft) Sektor. Zum anderen sollen die Schüler/ innen aber auch erkennen, dass die Einteilung in die Wirtschaftssektoren auf unterschiedlichen Begründungen beruhen kann. Während Fourastié die Kategorisierung der Sektoren insbesondere mit dem in ihnen jeweils möglichen technischen Fortschritt begründet, ist für Wolfe die Dominanz jeweils eines der drei Produktionsfaktoren (Boden, Kapital, Arbeit) relevant. Fisher wiederum begründet die Wirtschaftssektoren über die Preiselastizität ihrer Produkte (Pohl 1970). Mit diesem Schritt hätten die Schüler/innen somit ein Verständnis des Sektorenmodells erreicht, das wissenschaftspropädeutischen Standards entspricht.

\section{$4 \quad$ Methodik}

Die hier dargestellte Pilotstudie nutzt den ursprünglich aus dem japanischen Schulsystem stammenden Ansatz der lesson studies (Chassels 2009), der als eine
Form von action research betrachtet werden kann (Dudley 2014). Der Kern des Ansatzes besteht darin, dass ein Team von Lehrerinnen und Lehrern gemeinsam eine Unterrichtsstunde plant, die von einem Teammitglied unterrichtet wird, während die anderen Teammitglieder die Reaktionen einzelner Schüler/innen auf den Unterricht beobachten. Die Teamgröße beträgt meist zwischen drei und sechs Mitgliedern. Da alle Lehrer/innen an der Unterrichtplanung beteiligt waren, steht nicht die Unterrichtstätigkeit im Mittelpunkt der Beobachtung, sondern die Lerntätigkeit der Schüler/innen. Aus diesen Lernaktivitäten werden am Ende Schlüsse auf mögliche Verbesserungen der gemeinsamen Stundenplanung gezogen.

Die Arbeit des Teams aus Lehrerinnen und Lehrern erfolgt in der Regel in sieben Schritten (Cerbin \& Kopp 2006):

(a) Zunächst werden die zu erreichenden Lernziele definiert. Festgehalten werden soll, welche Konzepte oder Kompetenzen den Schülerinnen und Schülern vermittelt werden sollen.

(b) Auf der Grundlage der Lernziele wird gemeinsam eine Stunde geplant. Dabei ist es von Vorteil, wenn Lehrende mit unterschiedlichem Erfahrungshorizont zusammenarbeiten, z. B. Berufsanfänger/innen und Lehrende mit langer Berufserfahrung. Die Diskussion der geplanten Stunde soll auch dazu führen, dass die Lernbedürfnisse der Schüler/innen stärker ins Blickfeld rücken.

(c) Ist die Planung abgeschlossen, muss das Team entscheiden, wie es die Datenerhebung gestalten will. Die Beobachtung der Schüler/innen kann beispielsweise global für die ganze Klasse geschehen oder einzelne Teammitglieder beobachten jeweils nur eine/n Schüler/in, wobei die beobachteten Schüler/innen für das Leistungsspektrum der Klasse repräsentativ sein sollten. Zusätzlich zur Beobachtung können auch während des Unterrichts entstandene Arbeiten zur Auswertung herangezogen werden. Dazu muss allerdings vorher 
festgestellt werden, was aus den Arbeiten gelernt werden soll.

(d) Im nächsten Schritt erfolgt die eigentliche Unterrichtsbeobachtung.

(e) Hieran anschließend werden die erhobenen Daten analysiert und gemeinsam ausgewertet.

(f) Je nach dem Ergebnis der Datenauswertung wird die Unterrichtsstunde verändert und in der veränderten Form nochmals in einer anderen Klasse durchgeführt.

(g) Die Unterrichtsplanung und die Erhebung der lesson study werden in einer Form dokumentiert, die sie für andere nachvollziehbar und nutzbar machen.

$\mathrm{Da}$ es das Ziel der vorliegenden Studie war, die mögliche Progression im Kontext eines Sachthemas zu verfolgen, war die Durchführung mehrerer Stunden in verschiedenen Klassenstufen notwendig. Insgesamt wurden drei Unterrichtsstunden durchgeführt. Dabei lagen die beobachteten Klassen jeweils zwei Schuljahre auseinander, d.h. der Unterricht bezog sich auf eine 2., eine 4. und eine 6. Klasse.

Ziel der Stunde in der 2. Klasse war, den Schülerinnen und Schülern die Kategorisierung der Wirtschaftssektoren und ihre Konstruiertheit näher zu bringen. Dieses Ziel lässt sich durch eine Kombination der Basiskonzepte „Arbeit, Produktion, Konsum" (hier insbesondere der Produktion) und „Wahrnehmung und Darstellung" erreichen (vgl. BGBLA 2016). In der 4. Klasse sollte das Verständnis der Klassifikation erweitert werden um das Basiskonzept der räumlichen „Diversität und Disparität" (vgl. ebd.). $\mathrm{Zu}$ diesem Zweck wurde zunächst in das Modell des sektoralen Wandels eingeführt, um daran anschließend an Länderbeispielen zu prüfen, inwieweit sie diesem Modell entsprechen. In der 6. Klasse werden die Basiskonzepte aus der 2. Klasse erneut aufgenommen und vertieft. In diesem Kontext wird zunächst die Differenzierung des (nicht nur) in entwickelten Ländern oft über 70 Prozent ausmachenden Dienstleistungssektors diskutiert. Zudem sollte geprüft werden, ob und inwieweit Daten zu den Wirtschaftssektoren ausreichen, um den Entwicklungsstand eines Landes bestimmen zu können. Ziel war es, zu zeigen, dass unterschiedliche Zuordnungen nicht notwendig unterschiedliche Realitäten abbilden, sondern dass die Nutzung unterschiedlicher Modelle oder unterschiedlicher Datensätze zu unterschiedlichen Zuordnungen führen kann.

Die Unterrichtsbeobachtung wurde von jeweils drei Teammitgliedern durchgeführt, wobei jedes Mitglied eine/n Schüler/in beobachtet hat. Um die Ergebnisse möglichst gut vergleichbar zu machen, wurde ein Beobachtungsbogen erstellt, der als Leitfaden genutzt wurde. Besondere Aufmerksamkeit lag dabei darauf, wann ein/e Schüler/in dem Unterricht verständig folgte und wann er oder sie Schwierigkeiten hatte oder abgelenkt war, um aus den Reaktionen Rückschlüsse auf die Passgenauigkeit des Unterrichts ziehen zu können. Die Ergebnisse wurden von den einzelnen Beobachterinnen für die Auswertung zeitnah protokolliert. Ebenfalls für die Auswertung herangezogen wurden die Arbeiten, die die beobachteten Schüler/innen während der Stunde angefertigt hatten.

Alle drei Stunden wurden an zwei aufeinanderfolgenden Tagen durchgeführt. Im Anschluss an die letzte Stunde fand ein erstes Auswertungsgespräch statt, in dessen Verlauf die Eintragungen auf den Beobachtungsbögen verglichen wurden, ein zweites Gespräch folgte einige Wochen später. Die Stunde aus der 6. Klasse wurde daraufhin modifiziert, aber nicht noch einmal ausprobiert.

Die Ergebnisse der Beobachtungen sollen im nächsten Abschnitt differenziert dargestellt werden.

\section{Ergebnisse}

Um eine bessere Einordnung der Beobachtungen zu ermöglichen, wird im Folgenden jeweils erst kurz die geplante Stunde beschrieben. Danach werden die Reaktionen der drei beobachteten Schüler/innen dargestellt und interpretiert.

Bei der Planung der Unterrichtsstunde für die 2. Klasse ging das Lehrer/innen-Team davon aus, dass der Begriff „Wirtschaftssektoren“ den Schülerinnen und Schülern völlig unbekannt ist, da sie bisher kein explizites Unterrichtsthema waren und auch im Alltag selten thematisiert werden. Die Stunde wurde somit als grundlegende Einstiegsstunde in der Progression konzipiert. Insgesamt gliedert sich die Stunde in fünf Phasen: einen Einstieg über einen Bildimpuls, eine Erarbeitungsphase zur Kategorisierung verschiedener wirtschaftlicher Tätigkeiten, eine Problematisierungsphase zur Fokussierung von „Made in Austria“ auf den primären Sektor (vgl. Abb. 1 und 2), eine Sicherungsphase mittels Kahoot (internetbasierter Multiple-Choice-Wettbewerb - vgl. Kahoot) und eine Reflexionsphase zu den Produktionsschritten von der Kartoffelernte bis zum Pommes-Verkauf am Imbissstand.

Die beobachteten Reaktionen der Schüler/innen auf diese Phasen wiesen beträchtliche Unterschiede auf. Eine Schülerin beschäftigt sich überaus konzentriert mit dem in der zweiten Phase ausgeteilten Arbeitsblatt. Auch während der gemeinsamen Problematisierungsphase lässt sie sich kaum dabei stören, die gefundenen Kategorisierungen farblich zu kodieren. Lediglich in der Sicherungsphase wird ihre Aufmerksamkeit stärker Richtung Plenum gerichtet. Ganz 
anders verhält sich einer der beobachteten Schüler. Er beteiligt sich lebhaft und beantwortet besonders engagiert die komplexeren Fragen, etwa die nach der Herkunft dreier Produkte, die mit dem ÖsterreichLogo beworben wurden. Bei leichteren Fragen wirkt er eher abgelenkt. Auch die Beschäftigung mit dem Arbeitsblatt wird von vielen anderen Aktivitäten unterbrochen, sei es die Suche nach Stiften oder das Aufheben eines heruntergefallenen Blatts. Das Kahoot scheint diesen Schüler weniger methodisch als vielmehr inhaltlich zu motivieren. Der zweite beobachtete Schüler kann sowohl mit der Einzelarbeit in der Erarbeitungsphase als auch mit der gemeinsamen $\mathrm{Zu}$ sammenfassung gut umgehen. Er sucht allerdings des Öfteren Kontakt zum Nachbarn, entweder um ihm zu helfen oder gemeinsam Begriffe im Suchsel (Buchstabensalat, Wortsuchrätsel - vgl. Wikipedia) zu finden. Im deutlichen Unterschied zur sonstigen Aktivität bleibt dieser Schüler in der Problematisierungs- und der Reflexionsphase ruhig: Er konzentriert sich ganz auf das Zuhören.

Trotz der sehr unterschiedlichen Reaktionen konnten alle Schüler/innen dem Unterricht folgen und an verschiedenen Stellen valide Antworten geben. Die geplante Stunde scheint insoweit verschiedene Lernstile und Leistungsniveaus anzusprechen und jeder/jedem einen geeigneten Zugang zum Thema zu bieten.

Die Planung für die 4. Klasse sollte jedenfalls hypothetisch an die Inhalte der Stunde in der 2. Klasse anschließen. Da wir aber keine Längsschnittstudie durchgeführt haben und es sich bei der 4 . Klasse somit um komplett andere Schüler/innen handelte, mussten wir hoffen, dass die Klasse einen vergleichbaren Unterricht genossen hatte. Jedenfalls setzen eingeführte Lehrbücher der 3. Klasse bei Themen wie z. B. „Berufe im Wandel der Zeit" das Sektorenmodell ohne weitere Erklärung voraus: „Ein Großteil der Berufe ist im dritten Sektor angesiedelt, ..." (Mayrhofer, Posch $\&$ Reiter 2012). Tatsächlich reichte ein kurzgehaltener lehrerzentrierter Einstieg in das Thema aus, um das Vorwissen der Schüler/innen zu aktivieren. Aufbauend auf diesen Einstieg sollte der sektorale Wandel behandelt werden. Hierfür wurden vier Phasen geplant. In der ersten Phase sollten sich die Schüler/ innen den Wandel der beruflichen Situation über mehrere Generationen einer Familie erarbeiten. Anschließend wurde das Modell des sektoralen Wandels eingeführt, das in der Sicherungsphase am Beispiel von Mönchengladbach konkretisiert wurde. In der Reflexionsphase wurden stereotype Zuordnungen von Ländern mithilfe unerwarteter Beispiele (Agrarexporte der USA, Industrie in Liechtenstein, medizinische Dienstleistungen in Indien) hinterfragt und diskutiert.

Alle drei beobachteten Schüler/innen brauchten etwas Zeit, um einen Zugang zum Thema zu finden.
Eine Schülerin beginnt die Tabelle mit der Einteilung der drei Sektoren von der PowerPoint-Folie in ihr Heft zu übertragen. Dadurch verzögert sich der Beginn der Arbeit an der ersten Aufgabe, was im Laufe der Stunde zu immer neuen Verzögerungen führt. Trotzdem antwortet die Schülerin zu den jeweils ersten Teilen der Aufgabenstellungen korrekt. Erst in der Reflexionsphase lässt die Schülerin eine erhöhte Konzentration auf die tatsächlich gerade aktuelle Aufgabe erkennen. Deutlich eher findet der beobachtete Schüler seinen Weg in das Thema. Zwar wirkt auch er in der ersten und zweiten Phase noch eher müde, aber ab der Präsentation des Modells durch die Lehrperson ist eine ernsthafte passive und aktive Beteiligung festzustellen. Sie äußert sich zunächst im konzentrierten Zuhören und $\mathrm{ab}$ der Sicherungsphase auch in der intensiven Bearbeitung der Aufgabe sowie in mündlicher Beteiligung. Die zweite beobachtete Schülerin ist von Beginn an engagiert an der Erarbeitung beteiligt. Aber auch sie widmet ihre Aufmerksamkeit von Zeit zu Zeit anderen Schülerinnen oder Schülern, sei es, weil diese Lernenden sie von sich aus beeinflussen, sei es, weil ihr die Beobachtung der Reaktionen der anderen wichtig ist. Insgesamt fällt auf, dass sie sich bevorzugt bei komplexeren Fragen zu Wort meldet und meist valide Antworten gibt. Ihr gelingt es sogar, komplizierte Zusammenhänge für die anderen Lernenden verstehbar zu machen (Kommentar des angesprochenen Schülers: „Ach, jetzt versteh ich das“). In der Reflexionsphase äußert sie allerdings eher stereotype Vorstellungen bei der Beurteilung der Bilder.

Ähnlich wie in der ersten Gruppe lassen sich auch hier unterschiedliche Aktivitätsmuster ausmachen. Besonders unterschiedlich reagieren die beobachteten Schüler/innen in der Einstiegsphase: Während eine Schülerin den wiederholten Inhalt komplett notiert, zeigt sich der Schüler eher gelangweilt und die andere Schülerin testet ihr Wissen durch Teilnahme am Geschehen. Auffällig ist auch, dass die beiden Lernenden, die eher mühsam in den Unterricht finden, sich in der Sicherungs- und Reflexionsphase durch quantitativ und qualitativ gute Mitarbeit auszeichnen. Die Anfertigung von Notizen von dem wiederholten Stoff kann hier somit nicht als Hinweis darauf gelten, dass die Schülerin den Stundeninhalt insgesamt zu schwer finden könnte. Die andere Schülerin, die von Beginn an mitarbeitet und durch hilfreiche Antworten und Erläuterungen auffällt, tut sich dagegen gerade bei der Bewertung der angesprochenen Sektoren einzelner Länder deutlich schwerer.

In Hinblick auf die Progression von der Einführung der Wirtschaftssektoren als Kategorien zu einer Dynamisierung am Beispiel des Modells ist festzustellen, dass die Schüler/innen diesen Schritt weitgehend nachvollziehen konnten. 
In der 6. Klasse wurde die im Kontext der Progression besondere Herausforderung der gelungenen Einstiegsphase noch deutlicher. In der ursprünglichen Stundenplanung waren vier Phasen vorgesehen. In der Einstiegsphase sollte das - wiederum hypothetische Vorwissen durch die Erstellung einer Mind-Map aktiviert werden. Anschließend sollte die ursprüngliche Modellvorstellung differenziert werden, indem die Hinzufügung weiterer Sektoren diskutiert wird. In einer zweiten Erarbeitungsphase sollte thematisiert werden, wie die Daten, die zur Zuordnung einzelner Tätigkeiten zu den Kategorien des Modells notwendig sind, erhoben und verarbeitet werden und ob sie ausreichen um, wie im Modell vorgesehen, den Entwicklungsstand einzelner Länder zu bestimmen. Abschließend war eine kritische Reflexion von Modell und Datennutzung vorgesehen.

In der tatsächlichen Unterrichtssituation nahm die Einstiegsphase insbesondere durch die Zuordnung von durch die Schüler/innen selbst zu benennende Berufe deutlich mehr Zeit in Anspruch als ursprünglich geplant. Es entstanden bei einem Teil der Klasse unerwartete Seitengespräche, die Rückschlüsse darauf zuließen, was aus der Sicht der Schüler/innen das eigentlich Interessante an dem Thema wäre. Beispielsweise diskutierten sie konzentriert, ob Prostitution als Beruf gelten kann und wenn ja, welchem Sektor sie zuzuordnen wäre. Einige der beobachteten Schüler/innen waren Teil dieser Diskussionsgruppe, andere schienen von der Debatte eher gelangweilt zu sein. Durch diese Verzögerungen konnte nur mehr in Einzelarbeit und einem kurzem Plenumsgespräch eine Progression von fachlichen Inhalten - die Erweiterung des zugrundeliegenden Modellbegriffs durch die Einführung des Quartär- und Quintärsektors - umgesetzt werden. Das weit anspruchsvollere Ziel, eine Diskussion darüber anzuregen, inwieweit Daten zu den Wirtschaftssektoren ausreichen, um den Entwicklungsstand von Staaten zu bestimmen, konnte aus Zeitmangel in dieser Stunde nicht erreicht werden.

Trotz des unplanmäßigen Verlaufs der Stunde ergeben sich im Ablauf der Reaktionen einige Übereinstimmungen mit der zuvor beschriebenen 4. Klasse. Der beobachtete Schüler ist zwar von Beginn der Stunde an erkennbar bei der Sache, beteiligt sich aber nahezu nicht am Unterrichtsgeschehen. Stattdessen werden die Inhalte in einer kleinen Gruppe von benachbarten Schülerinnen und Schülern diskutiert. In dieser Gruppe entsteht auch die Diskussion darüber, ob Prostitution ein Beruf sei. Da die Diskussion sehr dominant wird, trägt sie erheblich zur Verlängerung der Einstiegsphase bei. In den beiden anschließenden Erarbeitungsphasen vertieft der Schüler sich zwar in die Texte, beteiligt sich aber nach wie vor nicht am Unterrichtsgeschehen. Ansprechpartner bleiben die
Nachbarn. Erst ganz am Ende der Stunde macht er durch intensiv genutzte Gestik deutlich, dass er den Ausführungen im Plenum folgt und folgen kann. Die erste beobachtete Schülerin holt noch während der Einstiegsphase ihre Arbeitsmaterialien aus dem Regal. Danach zeichnet sie das Tafelbild ab. Sie beteiligt sich zwar nicht am Unterrichtsgespräch, geht aber zur Tafel und ergänzt die Mind-Map. Sie wirkt dabei eher gelangweilt und müde. Die Diskussion um die Prostitution nimmt sie zwar wahr, fühlt sich aber nicht animiert, an ihr teilzunehmen. In den Erarbeitungsphasen wird sie deutlich aktiver, liest die Texte zügig und beantwortet die Fragen weitgehend korrekt. Die zweite beobachtete Schülerin ist zu Beginn engagierter: Sie schreibt ebenfalls mit, diskutiert mit ihrer Nachbarin und geht an die Tafel um die Mind-Map zu ergänzen. Am Unterrichtsgespräch beteiligt auch sie sich nicht. Ebenso zeigt sie kein Interesse an der Diskussion um die Prostitution. In dieser ungeplanten Diskussionsphase wirkt sie eher gelangweilt und teilnahmslos. Dies ändert sich in der anschließenden Erarbeitungsphase erneut: Hier liest sie die Texte konzentriert und zeigt eine deutliche Bereitschaft, sich an der mündlichen Zusammenfassung zu beteiligen. Nachdem sie mehrfach nicht drangenommen wurde, erlischt die Aufmerksamkeit zunehmend und sie wendet sich vermehrt den Kommunikationsbemühungen ihrer Nachbarin zu.

Der Schwachpunkt dieser Stunde liegt vor allem, aber nicht nur, in der Einstiegsphase: Hier werden die Schüler/innen auf einem zu niedrigen Niveau angesprochen, das jedenfalls bei einigen Schülerinnen und Schülern Raum für eigene, aber nicht unbedingt zielführende Phantasien lässt. Die sich daraus ergebende Diskussion verlängert den Einstieg, ohne dass alle anderen mitgenommen werden. In der nun zu kurzen Erarbeitungsphase erscheinen alle drei Lernenden motivierter. Sie deuten mit verschiedenen Gesten Verständnis an. Ein Rückfall in eher passive Verhaltensweisen lässt sich vor allem mit der fehlenden Berücksichtigung von Meldungen erklären. Insgesamt entsteht der Eindruck, dass der geplante Inhalt der Stunde eher „zu leicht“ als „zu schwer“ für die Schüler/innen war.

Auf der Grundlage dieser Erfahrung und Einschätzung ist die Planung für die Stunde für die 6. Klasse deutlich überarbeitet worden. Die Einführung wurde nicht mehr als einfache Wiederholung, sondern als erweiternde Wiederholung gestaltet. Dazu wird in Gruppen eine sorting exercise durchgeführt, in deren Verlauf abstrakte Konzepte wie etwa „arbeitsintensiv“, „Verarbeitung von Rohstoffen“ oder eben auch „Primärsektor" zu Kategorien zusammengefasst werden sollen. In den folgenden beiden Erarbeitungsphasen wird das Modell der Wirtschaftssektoren in zwei Rich- 
tungen differenziert: Zunächst wird die Erweiterung um den quartären und den quintären Sektor erläutert, anschließend liegt der Fokus auf verschiedenen Begründungen für die Ausweisung der Sektoren. In der Sicherungsphase wird der kritische Umgang mit dem Modell diskutiert. Mit dieser Planung wird dem Entwicklungsstand der Jugendlichen stärker Rechnung getragen: Sie langweilt sie nicht mit einer einfachen Wiederholung und sie erlaubt durch die erweiternde Wiederholung einen weiteren, theorieorientierten Schritt in der Progression.

Aus dem Verlauf der hier dargestellten lesson studies ergab sich der Rückschluss, dass der Phase des Unterrichtseinstiegs für eine gelingende Lernprogression besonderes Augenmerk geschenkt werden muss. Die Erhebung von Vorwissen als Ausgangs- und Anknüpfungspunkt für den weiterführenden Unterricht muss zielgerichtet und strukturiert erfolgen, um zu verhindern, dass sich Inhalte ständig wiederholen und Desinteresse entsteht. Darauf aufbauend können tatsächlich komplexere Denkprozesse angeleitet werden.

\section{Fazit}

Trotz der Existenz vieler unterschiedlicher Kompetenzmodelle sind Fragen nach der sinnvollen Gestaltung von Progressionen in Sachfächern noch weitgehend ungeklärt. Dies zeigt sich insbesondere auch an dem Versuch Kompetenzstufen für die financial literacy zu formulieren: Er scheitert an der Fokussierung auf deklaratives Wissen.

In der vorliegenden Studie wurde deswegen versucht die Progression über ein vertieftes konzeptuelles Verständnis zu definieren: von der Kategorisierung über den zeitlichen Wandel hin zu theoretischen Differenzierungen. Dieser Ansatz hat sich als überaus fruchtbar erwiesen. Er würde es auch erlauben, eine Progression über verschiedene Themen hinweg zu gestalten, etwa wenn Fragen der Kategorisierung am Beispiel der Produktionsfaktoren diskutiert werden, Fragen des zeitlichen Wandels am demographischen Übergang und Fragen der Differenzierung von Modellen am Beispiel der Wirtschaftssektoren. Um die konkreten Stufen einer themenübergreifenden Progression festzustellen, sind allerdings weitere Forschungen nötig.

In Bezug auf die unterrichtliche Umsetzung ist deutlich geworden, dass die einfache Wiederholung von zuvor behandelten Inhalten zeitraubend ist und oft zu Langeweile führt. Angemessener erscheinen erweiternde Wiederholungen, die zwar auf Vorwissen zurückgreifen, es aber in einen neuen Kontext stellen. Hierzu fehlen in der Literatur zu Unterrichtseinstiegen bisher ausreichende theoretische und praktische
Überlegungen, die den Lehrenden helfen könnten geeignete Einstiege zu konzipieren.

Eine entsprechende Forschung und Entwicklung würde es Schülerinnen und Schülern erlauben, ihre Fähigkeiten auf hohem Niveau weiterzuentwickeln.

\section{Dank}

Die Autorinnen bedanken sich bei der SoE der Alpen-Adria-Universität Klagenfurt für die finanzielle Förderung der Studie.

\section{Literatur}

Bagoly-Simó, P. \& A. Uhlenwinkel (2017): Germany: The need for rigorous conceptualization of progression. In: Muñiz Solari, O., M. Solem \& R. Boehm (Hrsg.): Learning Progressions in Geography Education. An International Perspective. Springer, Dordrecht/Heidelberg/ London/New York. S. 19-34.

Cerbin, W. \& B. Kopp (2006): Lesson Study as a Model for Building Pedagogical Knowledge and Improving Teaching. In: International Journal of Teaching and Learning in Higher Education 18(3). S. 250-257.

Chang, C.-H. (2017): Singapore: Curriculum Making and Geographical Learning Progressions. In: Muñiz Solari, O., M. Solem \& R. Boehm (Hrsg.): Learning Progressions in Geography Education. International Perspectives. Springer, Dordrecht/Heidelberg/London/New York. S. 111-123.

Chassels, C. (2009): Collaborative, Reflective, and Iterative. Japanese Lesson Study in an Initial Teacher Education Program: Benefits and Challenges. In: Canadian Journal of Education 32(4). S. 734-763.

Dudley, P. (2014): Lesson Study: a handbook. http://lessonstudy.co.uk/wp-content/uploads/2012/03/new-handbook-revisedMay14.pdf (27.03.2018).

Europarat (2001): Gemeinsamer Europäischer Referenzrahmen für Sprachen: Lernen, lehren, beurteilen. Langenscheidt, Berlin.

Greimel-Fuhrmann, B., M. Silgoner, R. Weber \& M. Taborsky (2016): Financial Literacy in Austria. In: Aprea, C., E. Wuttke, K. Breuer, N. K. Koh, P. Davis, B. GreimelFuhrmann \& J. S. Lopus (Hrsg.): International Handbook of Financial Literacy. Springer, Singapore. S. 251-262.

Haigner, S. D., S. Jenewein \& F. Wakolbinger (2016): Stiefkind Wirtschaftskunde. Eine Analyse von Schulbüchern: einseitig, falsch, tendenziös? Frank\&Frei, Wien.

Mayrhofer, G., R. Posch \& I. Reiter (2012): GEOprofi 3. Veritas, Linz. S. 108.

Muñiz Solari, O., M. Solem \& R. Boehm (2017): Preface. Building a Foundation for International Collaborative and Comparative Research on Geography Learning Pro- 
gressions. In: Muñiz Solari, O., M. Solem \& R. Boehm (Hrsg.): Learning Progressions in Geography Education. An International Perspective. Springer, Dordrecht/Heidelberg/London/New York. S. v-xiii.

OECD (2005): Definition und Auswahl von Schlüsselkompetenzen: Zusammenfassung. https://www.oecd. $\mathrm{org} /$ pisa/35693281.pdf (15.04.2018)

OECD (2013): PISA 2012 Assessment and Analytical Framework. Mathematics, Reading, Science, Problem Solving and Financial Literacy. OECD Publishing. https://www.oecd-ilibrary.org/docserver/9789264190511en.pdf (15.04.2018).

OECD (2014): PISA 2012 Results: Students and Money. Financial Literacy Skills for the 21st Century (Volume VI). OECD Publishing. http://dx.doi.org/10.1787/ 9789264208094-en (13.08.2018).

Pohl, H.-J. (1970): Kritik der Drei-Sektoren-Theorie. In: Mitteilungen aus der Arbeitsmarkt- und Berufsforschung 3(4). S. 313-325.

Robertson, M., J. Morgan \& J. Kriewaldt (2017): Australia and New Zealand. Do We Need to Understand ,Progress' Before We Can Develop Learning Progressions? In: Muñiz Solari, O., M. Solem \& R. Boehm (Hrsg.): Learning Progressions in Geography Education. An International Perspective. Springer, Dordrecht/Heidelberg/ London/New York. S. 1-17.

Schmidt-Wulffen, W. (1982): Allgemeine Geographie. In: Jander, L., W. Schramke \& H.-J. Wenzel (Hrsg.): Metzler Handbuch für den Geographieunterricht. Ein Leitfaden für Praxis und Ausbildung. Metzler Verlag, Stuttgart. S. 15-21.

Schultze, A. (1970): Allgemeine Geographie statt Länderkunde! Zugleich eine Fortsetzung der Diskussion um den exemplarischen Erdkundeunterricht. In: Geographische Rundschau 22(1). S. 1-10.

Solem, M. \& D. Lambert (2014): Researching Progress and Sophistication in Geography Learning: Taking a Critical Stance. In: Solem, M., N. Tu Huynh \& R. Boehm (Hrsg.): GeoProgressions. Learning Progressions for Maps, Geospatial Technology, and Spatial Thinking: A Research Handbook. AAG, Washington, DC. S. 61-69.
Stanat, P., R. Watermann, J. Baumert, E. Klieme, C. Artelt, M. Neubrand, M. Prenzel, U. Schiefele, W. Schneider, G. Schümer, K.-J. Tillmann \& M. Weiß (2002): Rückmeldung der PISA 2000-Ergebnisse an die beteiligten Schulen. Max-Planck-Institut für Bildungsforschung, Berlin.

Steegen, A., J. Coppenholle, A. Goemans \& L. Slegers (2017): Belgium. Learning Progressions in Geography Education: The Need for Definition. In: Muñiz Solari, O., M. Solem \& R. Boehm (Hrsg.): Learning Progressions in Geography Education. An International Perspective. Springer, Dordrecht/Heidelberg/London/New York. S. $125-138$.

Taylor, L. (2008): Key concepts and medium term planning. In: Teaching Geography 33(2). S. 50-54.

Uhlenwinkel, A. (2018): Ergebnisorientiert und interessengeleitet - Studien der Wirtschaftslobby zum GW-Unterricht. In: GW-Unterricht 150. S. 20-33.

Weeden, P. \& G. Butt (2009): Assessing Progress in Your Key Stage 3 Geography Curriculum. Sheffield, GA.

Willis, L. E. (2008): Against Financial-Literacy Education. In: Iowa Law Review 94(1). S. 197-285.

\section{Gesetzestexte}

BGBLA 2016_II_219 vom 9. August 2016. https://www. ris.bka.gv.at/ Dokumente/BgblAuth/BGBLA_2016_ II_219/BGBLA_2016_II_219.pdf (02.07.2017)

\section{Internet-Quellen}

Kahoot; abgerufen unter https://kahoot.com/

Kleine Zeitung vom 28.10.2016; abgerufen unter https:// www.kleinezeitung.at/wirtschaft/5109423/Weltspartagbei-Erste-Bank_Sprinkleranlage-ausgeloest_Kalte

Wikipedia: Suchsel; abgerufen unter https://de.wikipedia. org/wiki/Suchsel 